\title{
Cotrel-Dubousset Rods in Spinal Fractures
}

\author{
G. G. McBride, MD \\ Lucerne Spinal Center, 818 South Main Lane, Orlando, Florida 32801, USA.
}

\begin{abstract}
Summary
Cotrel-Dubousset instrumentation (CDI) has been gaining popularity in scoliosis surgery because of their improved rigidity which can obviate the need for a brace in most cases. Early results of this new system in scoliosis surgery have demonstrated a low pseudarthrosis and hardware complication rate. Because of these advantages, CDI was used to stabilise thoracolumbar fractures at the Lucerne Spinal Center. The results of 23 patients were reviewed with respect to completion of healing, change in neurological status, hardware complications, and follow-up radiographic parameters. Nine patients were braced and 14 were not braced post-operatively. Two elderly patients expired at 1 and 6 months following their injury from medical complications. Of the remaining 21 patients, all were evaluated at follow-up ranging from 6 to 28 months, mean 12.7 months. The evaluation revealed that all patients showed complete healing without significant neurological or hardware complications.
\end{abstract}

Key words: Cotrel-Dubousset instrumentation; Thoracolumbar fractures.

The treatment of unstable thoracolumbar fractures has evolved from body casting and recumbancy to early internal fixation and mobilisation over the past 30 years. Harrington instrumentation has become a standard accepted method of achieving sufficient stabilisation to allow patients to be mobilised within a few days of surgery in a body brace or cast (Yosipovitch et al., 1977; Dickson et al., 1978). However, even among experts, the hardware complication and pseudarthrosis rate may be as high as 27 per cent (Dickson et al., 1978).

Cotrel-Dubousset instrumentation has been gaining popularity in scoliosis surgery (Cotrel and Dubousset, 1985; Shufflebarger and Clark, 1986). Because of its increased rigidity and segmental fixation derived from multiple hook attachments and crosslinking (Johnson et al., 1986), scoliosis patients are allowed to be mobilised brace free following surgery in most cases. Other advantages of this spinal fixation system are its adaptability in contouring the rods to preserve 
Table I Treatment information

\begin{tabular}{|c|c|c|c|c|c|c|c|c|c|}
\hline \multirow[b]{2}{*}{$\begin{array}{l}\text { Init. } \\
\text { type }\end{array}$} & \multirow[b]{2}{*}{ Age } & & \multicolumn{5}{|c|}{ Fracture angle ${ }^{\star}$} \\
\hline & & $\begin{array}{l}\text { Level of } \\
\text { injury }\end{array}$ & $\begin{array}{l}\text { of injury } \\
\text { (degrees) }\end{array}$ & $\begin{array}{l}\text { Levels of } \\
\text { fixation }\end{array}$ & $\begin{array}{l}\text { Brace } \\
\text { type }\end{array}$ & $\overline{\text { init. }}$ & $\overline{\text { post-op. }}$ & $\overline{\text { final }}$ & $\begin{array}{c}\text { Follow-up } \\
\text { months }\end{array}$ \\
\hline E.G. & 26 & T3-4 & MVA & $\mathrm{T} 1-7(1)$ & HALO & 25 & 12 & 14 & 28 \\
\hline R.J. & 30 & L2 & Fall & T9-L5(1) & TLSO & 17 & 6 & 8 & 24 \\
\hline S.C. & 26 & $\mathrm{~L} 2-3 \mathrm{FXD} / \mathrm{L}$ & MVA & $\mathrm{T} 10-\mathrm{L} 4(2)$ & TLSO & 17 & -11 & -8 & 18 \\
\hline H.M. & 65 & L1 burst & Fall & T10-L4(1) & TLSO & 6 & 7 & 10 & 17 \\
\hline J.K. & 30 & L2 & Fall & T9-L4(2) & TLSO & 4 & 13 & 13 & 12 \\
\hline J.W. & 29 & L2 burst & Fall & T9-L3(1) & No & 14 & 3 & 16 & 17 \\
\hline J.B. & 81 & $\mathrm{~T} 12-\mathrm{L} 1$ & Fall & $\mathrm{T} 10-\mathrm{L} 4(2)$ & TLSO & -10 & -2 & 一 & - \\
\hline W.S. & 22 & T5-6FX D/L & Fall & $\mathrm{T} 2-\mathrm{T} 9(1)$ & No & 11 & 11 & 15 & 16 \\
\hline P.M. & 30 & T9-10 & Fall & $\mathrm{T} 2-\mathrm{T} 9(1)$ & No & 24 & 14 & 13 & 12 \\
\hline B.W. & 15 & T12-L1 FX & MVA & T9-L3(1) & No & 29 & -3 & 0 & 14 \\
\hline R.L. & 33 & L2 burst & Fall & $\mathrm{T} 11-\mathrm{L} 4(1)$ & No & 12 & 0 & 11 & 13 \\
\hline R.H. & 25 & $\mathrm{~T} 7, \mathrm{~T} 8$ & MCA & $\mathrm{T} 4-\mathrm{T} 12(1)$ & No & 32 & 21 & 25 & 13 \\
\hline J.T. & 31 & L2 burst & MVA & T11-L4(2) & No & 17 & 0 & -2 & 12 \\
\hline V.B. & 19 & L1 burst & MCA & T9-L4(1) & No & 22 & 11 & 5 & 10 \\
\hline R.A. & 31 & L1 burst & MVA? & T9-L3(1) & TLSO & 23 & 15 & 20 & 10 \\
\hline A.P. & 68 & L3 burst & Fall & T12-L5(3) & $\mathrm{C}-35$ & 10 & 0 & 5 & 10 \\
\hline H.B. & 79 & $\mathrm{~T} 12-\mathrm{L} 1$ & Fall & T9-L3(2) & TLSO & 10 & 9 & 4 & 4 \\
\hline P.S. & 26 & L2 & Fall & T10-L4(3) & No & 12 & 3 & 3 & 8 \\
\hline R.K. & 35 & $\mathrm{~T} 4-5 \mathrm{FX} \mathrm{D} / \mathrm{L}$ & MVA & $\mathrm{T} 1-9(1)$ & No & 15 & 16 & 15 & 9 \\
\hline R.W. & 18 & $\mathrm{~T} 6$ & MCA & $\mathrm{T} 2-11(1)$ & No & 33 & 19 & 28 & 6 \\
\hline W.M. & 25 & T4-5 & MVA & $\mathrm{T} 2-\mathrm{T} 9(2)$ & No & 25 & 10 & 11 & 6 \\
\hline R.K. & 36 & L3 & Fall & T12-L5(3) & No & 7 & -11 & -3 & 6 \\
\hline P.C. & 40 & L1 burst & Fall & T9-L4(1) & No & 11 & 6 & 5 & 6 \\
\hline
\end{tabular}

* $(+)$ Number indicates kyphosis, $(-)$ number indicates lordosis

the normal thoracic kyphosis or lumbar lordosis. It can also function as a distraction or compression system if needed. As a result of these advantages over the Harrington system, Cotrel-Dubousset instrumentation is believed to be a superior system for spine fracture fixation.

\section{Materials and Methods}

Twenty three consecutive patients who were treated for unstable thoracic or lumbar fractures with CDI were followed up prospectively from January 1986 to April 1988. The patients medical charts and radiographs were reviewed. Neurological assessment was made initially and at follow-up intervals using the method of Frankel (Frankel et al., 1969).

There were 23 patients, 19 male and 4 female whose ages ranged from 15 to 81 years, mean 37 years. Eleven patients were injured in falls, 10 in motor vehicle accidents, and 2 were injured by direct blows from objects. At the time of admission 7 patients were complete paraplegics, 6 were incomplete, and 8 were normal neurologically. Ten of the patients had associated injuries that included 8 extremity fractures, 1 skull and 1 sternal fracture. One of the 10 also sustained a ruptured spleen and kidney. There were 7 fracture dislocations from T4 to T11, 3 fracture dislocations involving T12 and L1, 1 fracture dislocation involving L2 and L3, and 12 burst fractures from L1 to L3. Details of their treatment are listed in Table I.

Two elderly patients (ages 79 and 80 ) had the pre-existing condition of advanced ankylosing spondylitis. These 2 patients expired at 1 and 6 months 
following their injury from unrelated medical complications. Of the remaining 21 patients, all were evaluated at follow-up ranging from 6 to 28 months, with a mean of 12.7 months.

All patients except 2 were treated within 3 weeks of their injury. One of these patients who sustained an L2 burst fracture was treated for progressive kyphosis following a failed anterior implant 33 months post-injury. The remaining patient was treated for progressing deformity following a failed Luque rod 7 months post-injury. Two of the 23 patients had a laminectomy with posterolateral decompression prior to their CDI procedure. Two patients with severe burst fractures and an incomplete neurological deficit underwent anterior decompression and fusion as a second stage. Indications for the anterior decompression have been previously elaborated (Bradford and McBride, 1984). However, each of these patients had residual stenosis of greater than 50 per cent of the saggital canal diameter and showed minimal improvement in neurological function.

\section{Instrumentation description}

The proper configuration for instrumentation of spinal fractures with CDI had not been developed at the beginning of this study. Therefore, suitable configurations for instrumenting thoracic and lumbar fractures were developed using the following guidelines:

1. Two rods were utilised as in the Harrington system, however they were connected near their upper and lower ends with a pair of dynamic transverse traction devices (DTT) so that a rectangular configuration is created.

2. At least three normal vertebra above and two below the fracture site were spanned by the instrumentation.

3. Hooks are attached to the spine at two different sites above and two sites below the fracture site.

4. Patients having laminectomy and those who were complete paraplegics had slightly longer instrumentation and fusion than those who were intact or incomplete.

Using these concepts above, three configurations of CDI were utilised. The first configuration, designated as Type I, uses two pairs of hooks above and two pairs below the injury level in a distraction mode (Fig. 1, Fig. 2). This was used in 14 of the cases where there was a fracture dislocation component with anterior bony comminution (Fig. 3). The use of the Type I configuration also requires an intact anterior longitudinal ligament.

The second configuration, designated as Type II, uses the same number of hooks above and below the injury level as Type I but they are reversed into a compression mode (Fig. 4). This was used in 6 cases where there was a significant distraction component or a Chance type fracture and the posterior portion of the vertebral body (middle column) was intact. Another potential indication is a severely comminuted fracture or dislocation where the anterior longitudinal ligament is ruptured.

Type III is similar to Type I but the lowest pair of hooks are reversed so 
TYPE I

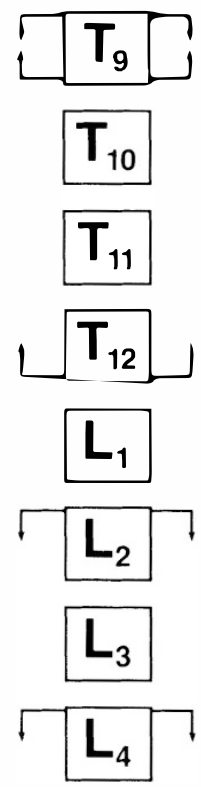

Figure 1 Diagram of Type I configuration showing hook placement for a fracture dislocation.

TYPE I

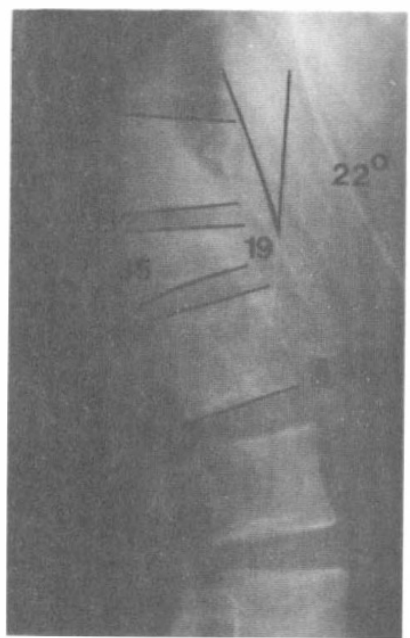

PRE-OP

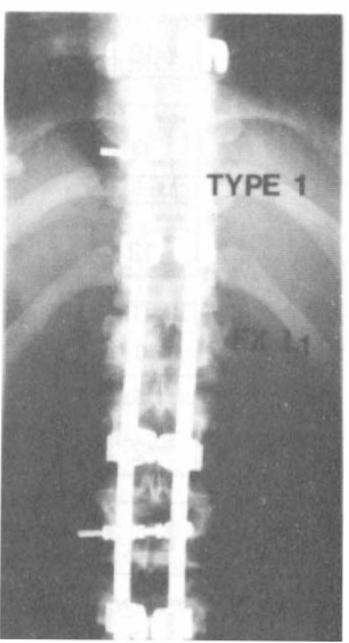

POST-OP

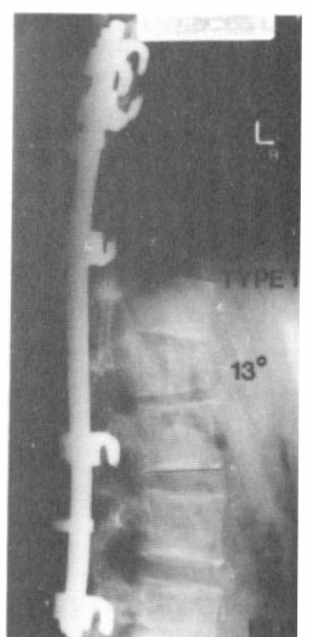

POST-OP

Figure 2 Composite radiograph of a 19-year-old male who sustained a burst fracture of L1, pre-operative and post-operative radiographs. 
TYPE II

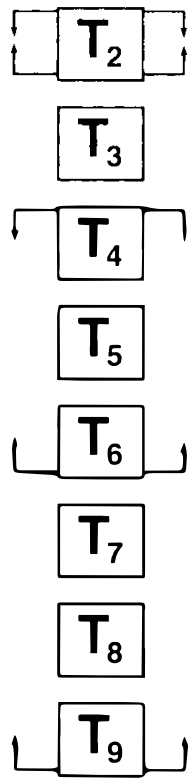

Figure 3 Diagram of Type II hook configuration for a flexion distaction injury, or when the anterior longitudinal ligament is not intact.

TYPE III

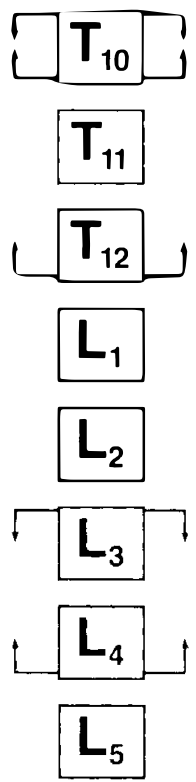

Figure 4 Diagram of Type III hook configuration used to maintain for a burst fracture of a mid-lumbar segment. 


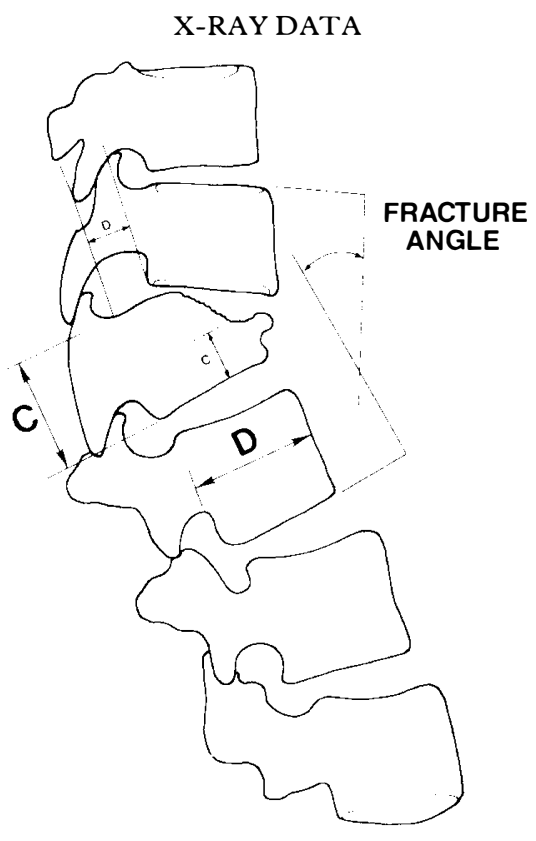

Figure 5 Analysis of radiographic data. Determination of the fracture angle is shown. Per cent compression is ${ }_{0}^{\circ}$ Comp. $=100-\mathrm{c} / \mathrm{C}$. Per cent displacement is ${ }_{0}^{\circ} \mathrm{Displ}=\mathrm{d} / \mathrm{D} \times 100$.

that the lower pair locks against the next pair above (Fig. 5). This configuration was utilised for 3 cases of mid-lumbar fractures where lordosis needed to be maintained and was developed by another investigator (Bridwell, 1986). It can also be used for more common thoracolumbar fractures but Type I is preferred.

The uppermost hooks in Types I to III were designed to couple with additional transverse process hooks to create a 'claw' attachment on the uppermost vertebra for additional stability (Figs 1-5). Standard surgical exposure and fusion techniques were employed (Flesch et al., 1977). Iliac crest graft was used in all cases. All levels instrumented were fused except when the fusion extended to L5. In those cases fusion was carried down to L4 and recommendations were made to remove the hardware at 1 year. Spinal evoke potential monitoring was utilized for incomplete or intact cases.

\section{Post-operative treatment}

The patients were treated without a post-operative brace if they met the following criteria: (1) absence of osteoporosis; (2) lack of additional spinal fractures; (3) anticipated good compliance with post-operative limitations with close follow-up. Accordingly 9 patients were braced and 14 were not braced following surgery. Those patients who were not braced were restricted from excessive bending and stooping and heavy lifting for 3 months. Those who were not braced were allowed to sit or stand, shower and swim as soon as they were able. 
Additionally, they were allowed to begin light exercise classes and practice dressing and transfer skills within 1 to 2 weeks of surgery. They were discharged as soon as they were functionally independent and all necessary home modifications were made.

\section{Radiographic analysis}

The pre-operative, post-operative, and follow-up radiographs were analysed with respect to fracture angle, per cent of compression, and fracture displacement. Fracture angle is defined as the angle formed by the perpendicular projections from two lines drawn parallel to the endplates of the adjacent vertebra above and below the fracture level. Per cent of compression is the ratio of the distance in millimetres of the front of a compressed vertebra compared to the posterior portion of the body and subtracted from 100. If more than one level was involved, then the most severely damaged level was measured. These results were tabulated for analysis.

The per cent displacement is defined as the ratio of the distance displaced in millimetres divided by the anterior-posterior dimension and multiplied by 100. All measurements were made on the lateral radiograph and tabulated for analysis.

Assessment of fusion was performed from standard A-P and lateral radiographs. Oblique radiographs were utilised in some cases to assess lumbar facet joint fusion. Special attention was made to observe any evidence of hardware migration or loosening and pseudarthrosis development. Solid union was determined by the formation of bone lateral and posterior to the rods along with the lack of any significant hardware displacement or loss of reduction. Many of these patients had relatively short follow-up which made only preliminary assessments possible.

Post-operative CT scans were performed in only those cases with incomplete neurological deficits that failed to improve neurologically. Although improvement in canal diameter was generally noted, there was not sufficient data for meaningful conclusions.

\section{Results}

Twenty one of the patients returned for follow-up. Two male patients, aged 79 and 81 years, expired from unrelated medical causes. One patient expired from a stroke and the other expired from general medical deterioration, 6 weeks and 6 months respectively following surgery. Each of these patients had severe ankylosing spondylitis and had been injured by relatively minor falls at home. Of the remaining 21 patients, all were showing signs of solid union at follow up. Complications are listed in Table II. There were no wound infections, and no instances of rod breakage or obvious loosening of hooks occurred. There was 1 case of slight hook migration and 1 case of diffuse rod pain not requiring medication. Two other patients had their rods removed at 16 months post-operatively because of persistent pain over a hook site. No patient had an increased neurological deficit following CDI. 
Table II Complications

\begin{tabular}{|c|c|c|}
\hline Major type & Number & Comment \\
\hline Infection & 0 & $\begin{array}{l}\text { Wound only, excludes } \\
\text { urinary }\end{array}$ \\
\hline Rod breakage or gross & 0 & \\
\hline loosening & 0 & \\
\hline Pseuarthrosis & 0 & \\
\hline Increased neuro deficit & 0 & Post-op. or follow-up \\
\hline Death & 2 & $\begin{array}{l}\text { Elderly patients of } \\
\text { unrelated medical causes }\end{array}$ \\
\hline \multicolumn{3}{|l|}{ Minor type } \\
\hline Slight hook migration & 1 & \\
\hline Diffuse rod pain & 1 & \\
\hline Rods removed & 2 & $\begin{array}{l}\text { Each at } 16 \text { months } \\
\text { post-op. for hook site pain }\end{array}$ \\
\hline
\end{tabular}

Table III Neurological status *

\begin{tabular}{llll}
\hline Initial status & Number & Post-op. & Follow-up \\
\hline Grade A & 7 & All A & All A \\
Grade B & 2 & B, B & D, D \\
Grade C & 3 & C, C, C & D, D, D \\
Grade D & 1 & D & E \\
Grade E & 8 & All E & All E \\
\hline
\end{tabular}

^Method of Frankel for 21 cases at follow-up.

The neurological status of the patients initially, and at the time of follow-up, is listed in Table III. There were 7 patients who remained complete paraplegics following surgery. Of the 6 incomplete injuries, all improved with 1 becoming normal. All patients who were normal neurologically remained unimpaired.

Two cases involving burst fractures with initially good reductions showed considerable fracture settling without an increase in fracture angulation over the follow-up period. One had a Type II, and the other a Type III fixation. Neither were braced post-operatively, and the settling did not affect the patient's neurological status or function.

The radiographic analysis showing the means of the pre-operative and postoperative fracture angulation, per cent compression and displacement is listed in Table IV. The per cent displacement data includes only those 8 cases of fracture dislocations or burst fractures with greater than $15^{\circ}$ o displacement.

\section{Discussion}

The goals of spinal instrumentation are to restore anatomical alignment and provide rigid stability to allow early mobilisation. A wide variation of injury pattern exists such as flexion-distraction and compression injuries, burst fractures, and flexion-rotation injuries. The importance of maintaining normal saggital plane alignment, or in other words, the normal thoracic kyphosis and lumbar lordosis is becoming an increasingly accepted requirement for spinal fixation systems. Harrington instrumentation cannot always meet these goals. 
Table IV Radiographic analysis

\begin{tabular}{lccc}
\hline Parameter & Initial & Post-op. & Follow-up \\
\hline Fracture angle (degrees) & 17 & 7 & 10 \\
Per cent compression & $37^{\circ}{ }^{\circ}$ & $22^{\circ}{ }^{\circ}$ & $26^{\circ}{ }^{\circ}$ \\
Per cent displacement & $27^{\circ}$ & $6^{\circ}{ }_{0}$ & $6^{\circ}$ \\
\hline
\end{tabular}

*Includes only those 8 cases with $15^{\circ}$ o or more initial displacement

Furthermore, the Harrington system has a significant pseudarthrosis and hardware complication rate as high as $27^{\circ}$, even in expert hands (Dickson et al., 1978).

A cast or polypropylene brace is usually required post-operatively after using the Harrington system ranging from 3 to 6 months (Flesch et al., 1977; Dickson et al., 1978). In a paraplegic individual, a post-operative brace or cast is an undesirable necessity that often leads to pressure sores of the skin and allows only basic upright activity in a wheelchair. On the other hand, with the CotrelDubousset system, it is often possible within 2 to 3 weeks for a patient to begin even advanced skill training such as bathing, dressing, self-catheterisation, bowel programmes, transfers and swimming without being hindered by a cumbersome cast or brace. Any delay in the rehabilitative process may not only have its psychological toll but may represent a significantly higher financial cost.

With all of these advantages with the CDI, it is hard to find many criticisms. It is definitely more expensive when compared to the Harrington system, but this is probably negated when the cost of a post-operative orthosis is included with Harrington rods. In addition, CDI is technically more difficult and time consuming to insert and may not be suitable when other procedures are contemplated in surgically unstable or polytrauma patients. There is a definite 'learning curve' with an increase in the surgical time which diminishes after the first 5 to 10 cases. For the casual spine surgeon or in a situation with an unstable or polytrauma patient the Harrington rod may be a better system. Also, the hardware is somewhat more prominent than the Harrington system and may require more removals in the long term. The average follow-up time in this investigation of 12.7 months is relatively short and the complication rate may be higher during a longer evaluation period.

The post-operative reduction achieved with the CDI system appears comparable to other stabilisation methods. Fracture angulation, displacement, and degree of fracture compression was greatly improved and well maintained in the majority of cases at follow-up review. Only 2 cases involving burst fractures showed increased settling but had minimal change in fracture angulation and no effect on neurological outcome.

The length of instrumentation in this series often averaged 1 to 3 levels longer than that reported with the Harrington system. In some cases associated laminectomy (of doubtful benefit) or the presence of adjacent fractured posterior elements dictated longer instrumentation. Also, technical difficulties in the placement of hooks at adjacent levels required spanning an additional level or two with the rods. Efforts are currently in progress to limit the instrumentation levels to 5 or 6 with slight modifications of the three types of configurations. 


\section{Conclusions}

In summary, Cotrel-Dubousset instrumentation appears to be a better system for spinal stabilisation because of its greater rigidity and more rapid patient mobilisation without the need for post-operative brace in most cases. It seems to be more adaptable than the Harrington system in a wide variety of spinal injuries. Using more hook attachment sites achieves a type of 'segmental' fixation that seems to result in better healing with a lower complication rate.

\section{References}

Cotrel Y, Dubousset J 1985 New segmental posterior instrumentation of the spine. Ortho Trans 9:118.

BRADFORD DS, MCBRIDE GG 1986 Delayed anterior decompression in thoracolumbar fractures. Clinical Orthopaedics and Related Research 186:75-84.

BRIDWELL KH 1986 Spinal fusion with Cotrel-Dubousset instrumentation and iliac bone graft. Video fournal of Orthopedics 1:2.

Dickson JH, HARRINGTON PR, ERWIN WD 1978 Results of reduction and stabilisation of the severely fractured thoracic and lumbar spine. fournal of Bone and foint Surgery 60A:799-805.

Flesch JR, LeIder LL, ERICKSON DL, CHOU, SN, BRAdFord DS 1977 Harrington instrumentation and spine fusion for unstable fractures and fracture dislocations of the thoracic and lumbar spine. Fournal of Bone and foint Surgery 59A:143-150.

FRANKel HL, HANCOCK DO, Hyslop G et al. The value of postural reduction in the initial management of closed injuries of the spine with paraplegia and tetraplegia. Paraplegia 7:179188.

Johnson CE, Ashman RB, CoRin JD 1986. The mechanical effects of cross-linking rods in Cotrel-Dubousset instrumentation. Scoliosis Research Society presentation.

Shufflebarger HL, Clark C 1986 Cotrel-Dubousset instrumentation in adolescent idiopathic scoliosis. Scoliosis Research Society presentation.

Yosipovitch Z, RoBIN GC, MANkin M 1977 Open reduction of unstable thoracolumbar spinal injuries and fixation with Harrington rods. fournal of Bone and foint Surgery 59A:1003-1014. 